\title{
Strategy-dependent changes in memory: Effects on behavior and brain activity
}

\author{
NICOLE K. SPEER, LARRY L. JACOBY, and TODD S. BRAVER \\ Washington University, St. Louis, Missouri
}

\begin{abstract}
In the present study, an implicit strategy manipulation was used to explore the contribution of memory strategy to brain activationand behavioral performance. Participants were biased to use either a shortterm (maintenance-focused) or long-term (retrieval-focused)memory strategy within a single memory task through manipulation of task context. In comparing directly matched trials across the different task contexts, we observed clear changes in both behavioral performance and brain activity across a network of regions located primarily within lateral and medial frontal cortex. These effects of the memory strategy manipulation suggest that when a retrieval-focused strategy is induced, mnemonic processes are preferentiallyengaged during the encoding period. In contrast, when a maintenance-focused strategy is induced, mnemonic processes are preferentially engaged during the delay and response periods. Taken together, the results imply that covert cognitive strategies play an important role in modulating brain activation and behavior during memory tasks.
\end{abstract}

Psychological research has a long history of dividing short-term memory (STM) and long-term memory (LTM; Atkinson \& Shiffrin, 1971; James, 1983; Waugh \& Norman, 1965). This separation has been supported by studies on the neural substrates of the memory processes engaged during the performance of STM and LTM tasks. Convergent results from single-cell neurophysiology, neuropsychology, and neuroimaging studies have suggested that STM involves storage through sustained neural activity in discrete brain regions, with the lateral prefrontal cortex (PFC) appearing to serve a critical role in this process (Barch et al., 1997; Cabeza \& Nyberg, 2000; Cohen et al., 1997; Courtney, Ungerleider, Keil, \& Haxby, 1997; D’Esposito et al., 1998; Fuster, 1989; Goldman-Rakic, 1987; Owen, 1997; Petrides \& Milner, 1982; Smith \& Jonides, 1999). In contrast, studies of LTM have emphasized the formation of more permanent episodic traces through mechanisms of associative (i.e., synaptic) strength and have focused on the hippocampus and the medial temporal lobes as key structures mediating LTM processes (Eldridge, Knowlton, Furmanski, Bookheimer, \& Engel, 2000; Mishkin, 1982; Scoville \& Milner, 1957; Vargha-Khadem et al., 1997; Zola-Morgan, Squire, \& Amaral, 1986).

The strongest support for the division of neural systems for STM and LTM in humans comes from the amnesia literature. Scoville and Milner (1957) originally discovered

This research was supported by National Institute of Mental Health Grant RO3 MH61615 to T.S.B. as well as by a National Science Foundation Graduate Research Fellowship to N.K.S. We thank Susan Bongiolatti and Mitch Dornfeld for help in data collection and Deanna Barch, Jeremy Reynolds, Jeffrey Zacks, and three anonymous reviewers for helpful comments on the manuscript. Correspondence concerning this article should be addressed to T. S. Braver, Department of Psychology, Campus Box 1125, Washington University, St. Louis, MO 63130 (email: tbraver@artsci.wustl.edu). that resecting the medial temporal lobe led to profound memory impairments. In later studies, it was found that this inability to remember information was present only with relatively long delays between study and test; retention of information over relatively short delays (e.g., digit span) was intact (Shimamura, Janowsky, \& Squire, 1991). These data were taken as evidence that the processes involved in long-term retention have different neural correlates than the processes involved in short-term retention. This pattern of memory deficits is in contrast with that observed following frontal lobe lesions, in which retention over short delays is more impaired than retention over long delays (Shimamura et al., 1991). Taken together, these data argue for a strict division of STM and LTM systems in the brain.

However, with the explosion of neuroimaging research in the last decade, this dichotomous picture of STM and LTM in humans has become more complex. It is now clear that the lateral PFC is reliably activated during the performance of LTM tasks (Brewer, Zhao, Glover, \& Gabrieli, 1998; Buckner, 1996; Buckner \& Koutstaal, 1998; Cabeza \& Nyberg, 2000; Wagner, Schacter, et al., 1998), and recent studies have found hippocampal activity during the performance of STM tasks (Cabeza, Dolcos, Graham, \& Nyberg, 2002; Ranganath \& D'Esposito, 2001).

One possible explanation for this mixed pattern of activity is that both STM and LTM processes are involved in the performance of a given memory task regardless of whether the task is characterized as an STM task or as an LTM task. That is, the mnemonic processes that contribute to long-term retention may operate in STM tasks, and the processes that contribute to short-term retention may operate in LTM tasks, leading to overlapping patterns of brain activation during STM and LTM task performance. This explanation is consistent with the views of early memory theorists, who acknowledged the contribution of LTM processes to STM tasks (Waugh \& Norman, 1965), 
with STM and LTM tasks differentiated on the basis of the amount of information stored and the duration of retention, and STM and LTM processes differentiated on the basis of the mnemonic mechanism or strategy that gives rise to task performance (Engle \& Oransky, 1999). Additional evidence for the idea that multiple mnemonic processes may contribute to performance in a single memory task comes from the amnesia literature: Amnesic patients show more forgetting than controls do during the primacy portion of the serial position curve (Capitani, Della Sala, Logie, \& Spinnler, 1992; Carlesimo, Sabbadini, Fadda, \& Caltagirone, 1995), whereas the recency portion of the curve remains intact.

Given that a single memory task most likely engages more than one type of memory process, studying differences in memory processes may be more fundamental than studying differences in memory tasks (Jacoby, 1991). However, in order to study differences in memory processes, the processes must be examined within the same memory task. Behavioral studies of memory have shown that the experimental design can selectively influence the nature of the contribution provided by multiple memory processes (e.g., Jacoby \& Kelley, 1991). The results of these studies demonstrate that it is possible to study multiple memory processes within a single memory task and suggest that with the use of more sophisticated experimental designs, the contribution of individual brain regions to the mnemonic processes underlying STM and LTM task performance may become clearer.

Recent neuroimaging studies of STM and LTM tasks have made progress in identifying the contributions of different brain regions to STM and LTM processes through comparisons of brain activation during both types of tasks, both within and across studies (Braver et al., 2001; Cabeza et al., 2002; Cabeza \& Nyberg, 2000; Nyberg, Forkstam, Petersson, Cabeza, \& Ingvar, 2002; Wagner, 1999; see Cabeza \& Nyberg, 2002, for a review). Acrossstudies comparisons have typically focused on identifying the different regions involved in STM and LTM task performance, whereas the more recent within-studies comparisons have focused on identifying different patterns of activation in response to STM and/or LTM tasks within the same regions. Although identical or nearly identical perceptual conditions were used in these studies, the explicit goals varied with each task. That is, whereas the perceptual input was similar in each task, the task demands, such as memory load, retention interval, and duration of encoding and retrieval phases, were very different. These prior neuroimaging studies have produced informative results in terms of demonstrating overall similarities and differences in regional activation during STM and LTM tasks (Braver et al., 2001; Cabeza et al., 2002; Nyberg et al., 2002). However, it is not clear whether these differences reflect shifts in the mnemonic processes engaged to perform the tasks or, instead, reflect a reaction to the change in explicit task requirements. The only way to determine clearly how a particular region might differentially contribute to STM and LTM task performance is by varying the involvement of STM and LTM processes while holding explicit task requirements constant.

One promising approach to separating task requirements from memory processes is to assume that the engagement of STM and LTM processes reflects strategic biases in memory usage. Although STM processes, such as maintenance rehearsal, and LTM processes, such as elaborative encoding, may both operate within a given memory task, participants may be able to regulate the degree to which each process contributes to task performance. On the basis of this assumption, it might be possible to influence an individual's bias toward one memory process over the other or to bias the temporal dynamics of the memory process by manipulating subtle contextual factors of the task environment while holding all other aspects of the task constant. Evidence that such contextual manipulations can differentially influence performance on otherwise identical memory tasks (i.e., tasks with the same explicit memory requirements) was observed in the earlier memory literature (Crowder, 1969), but has not been a prominent focus of recent work, especially within the neuroimaging community.

In this study, we attempted to influence the degree to which STM and LTM processes operate within a single memory task in order to explore the patterns of activation associated with changes in mnemonic strategy. The participants in this study performed two blocks of a memory task that required retaining a list of words over a brief delay and responding to a test item (probe word) to assess the participants' memory for the words in the list (see Figure 1). The difference between the two blocks was the expected number of words to be remembered. In the shortlist block, on average fewer than four words had to be remembered, whereas in the long-list block, on average more than eight words had to be remembered. We hypothesized that if the expected number of items to be remembered during a memory task were minimal, the participants would be biased toward an STM strategy (e.g., one involving active maintenance and articulatory rehearsal). In contrast, if the expected number of to-beremembered items was relatively high, the participants' bias would shift toward an LTM strategy (e.g., one involving elaborative encoding and cued retrieval; cf. Jacoby \& Bartz, 1972). In order to control for the differences in cognitive demands across task blocks, some of the trials within each block were identically matched on all task characteristics and requirements (i.e., memory load, retention interval, and the duration of encoding and retrieval periods). Thus, although tasks and processes were confounded across the short-list and long-list task blocks, by comparing performance on the explicitly matched trials embedded within each task block, we were able to observe effects of maintenance-focused and retrieval-focused memory strategies on behavior and brain activity.

The main difference between these two strategies was hypothesized to be the relative engagement of mnemonic processing mechanisms at different points of the trial. In a maintenance-focused strategy, the mnemonic processes were 


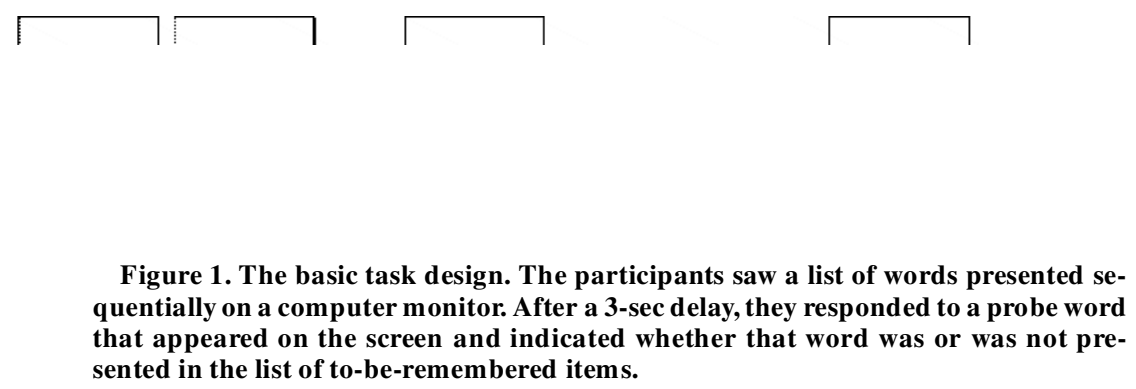

predicted to be preferentially engaged during the delay and response periods. The participants should have been able to actively rehearse the words in the list and would have done so in anticipation of quickly selecting a response to the probe word. When the probe word was presented, a search through the active representation of list items would result in a comparison between the probe word against the stored information to determine whether or not the probe word matched a maintained item. Conversely, in a retrieval-focused strategy, mnemonic processes were predicted to be preferentially engaged during the encoding period to ensure that a relatively robust memory trace would be formed for each item. The presentation of the probe word would then serve as a retrieval cue that could guide a relatively effortless retrospective search of such traces.

On the basis of the results of similar studies involving maintenance-focused strategies (Burrows \& Okada, 1975; Sternberg, 1966, 1967), response times (RTs) and accurate performance in responding to the probe word were expected to increase in a linear fashion with increases in list length during the short-list task block. However, there is no reason to expect a linear increase when participants are using a retrieval-focused strategy. Furthermore, if participants actively rehearse the words in the list during the short-list block, there should be less chance of forgetting the words over the delay. In contrast, if participants engage in a retrieval-focused strategy, they would be expected to require more processing of the probe word than if they were engaged in a maintenance-focused strategy. These differences in processing the probe word should be apparent when later memory for the probe words from the different task blocks is compared: Participants should show better delayed recognition of probe words seen in the long-list task block than of those seen in the short-list task block. Note that the fact that the probe word is processed more deeply in the long-list task block than in the shortlist task block does not imply that more memory resources are devoted to the response in the long-list task than in the short-list task. It is hypothesized that in the short-list task participants are actively rehearsing the list of words to determine whether one of the words in active memory matches the probe word, a process that surely requires a great deal of mnemonic resources. However, in the short-list task this effort is focused on the contents of active memory rather than on the probe word itself (which is why these strategies have been described as "maintenance-focused" and "retrieval-focused," respectively).
With regard to brain activity, we expected that the two task blocks would activate similar brain regions. That is, regions showing task-related activation in response to one task block were expected to show task-related activation in response to the other task block. This expectation was based on the similarity of the task requirements as well as on the results of similar studies in which a high degree of overlap in STM and LTM task-related activation was observed (e.g., Ranganath, Johnson, \& D'Esposito, 2003). However, we also expected that when we compared the patterns of brain activity on the identically matched trials, we would identify a subset of brain regions that reflected general shifts in memory strategies.

We expected to identify strategy-sensitive activation in brain regions thought to be involved with processes related to the strategic control of memory, such as elaborative encoding (e.g., left inferior PFC; Buckner, Kelley, \& Petersen, 1999), articulatory rehearsal (e.g., supplementary motor area; Chein \& Fiez, 2001), and episodic retrieval (e.g., anterior PFC; LePage, Ghaffar, Nyberg, \& Tulving, 2000). We predicted that these brain regions would show differences in activity during the course of the trial that would be consistent with preferential engagement of different mnemonic processes. For example, we predicted that brain regions supporting articulatory rehearsal would be preferentially engaged during the latter part of the trial in the short-list condition, in accordance with a maintenancefocused strategy. In contrast, in the long-list condition such regions might be preferentially engaged during the initial part of the trial, to facilitate elaborative encoding for the purposes of later retrieval.

We predicted that most strategy-sensitive regions would be found in the PFC, given the numerous findings of frontal involvement in LTM and STM tasks. Although many other nonfrontal regions are reliably activated in STM (e.g., parietal cortex) and LTM (e.g., medial temporal lobe) tasks, it is primarily the PFC that is thought to be involved in the strategic control of mnemonic processes.

\section{METHOD}

\section{Participants}

Sixty-five adults between the ages of 18 and 31 years (36 women, mean age $=20.5$ years) volunteered to participate in this study. Twenty-five of these participants ( 12 women, mean age $=21.0$ years) performed the study in the scanner. The participants who were scanned received a cash stipend, whereas the remaining 40 participants (24 women, mean age $=19.6$ years) performed the study for course 
credit. Informed consent was obtained in accordance with guidelines set by the Human Subjects Committee at Washington University.

\section{Stimulus Materials}

Two blocks of a memory task were used to induce STM and LTM strategies. The task required the participants to maintain a list of words in memory over a brief delay and to respond to a probe word to indicate whether or not it matched one of the words from the list. For the participants who performed the study outside the scanner, a yes/no recognition memory test was administered at the end of the session to assess memory for nontarget probe words that occurred in the word span tasks. A 10-15-min personality test served as a filler task between the word span tasks and the recognition memory task for these participants. The stimuli for both blocks of the memory task as well as for the recognition task consisted of one- and twosyllable English nouns randomly selected from a stimulus pool of 1,120 words obtained through the MRC Psycholinguistic Database (Wilson, 1988). The words in the pool had a mean Kučera and Francis (1967) written frequency rating of 43 and were between four and six letters long. The short-list memory block used 80 lists of 1, 2, 3, 4,5 (each length contributing to $15 \%$ of the trials in the block), and 6 (contributing to $25 \%$ of the trials in the block) words, whereas the long-list memory block used 80 lists of $6(25 \%), 7,8,9,10$, or 11 (15\% each) words. Stimulus presentation, timing, and response collection were controlled by PsyScope software (Cohen, MacWhinney, Flatt, \& Provost, 1993) running on an Apple PowerMac G3 computer. All words in the study were presented individually, centered on the computer monitor (Apple StudioDisplay, $43 \mathrm{~cm}$ in diameter) in 36-point Times New Roman font, and responses to probe words in all tasks were collected using the PsyScope Button Box.

\section{Procedure}

Memory tasks. The order of the short-list and long-list blocks was counterbalanced across participants, such that half of the participants completed the short-list block first followed by the long-list block, whereas the other half of the participants completed the longlist block followed by the short-list block. The participants were instructed to maintain either a relatively short list of words (short-list block) or a relatively long list of words (long-list block) over a brief delay, after which they would respond to a probe word and indicate whether the word matched or did not match one of the words in the list (see Figure 1). A trial was defined as the presentation of a list of words, the delay period, and the response interval. Each block consisted of 80 trials separated into two sets of 40 trials (behavioral study), two runs of two 20-trial sets (short-list task, fMRI study), or four runs of two 10-trial sets (long-list task, fMRI study). The participants were given a break between sets within each run in order to decrease fatigue and maximize task performance. The procedure was identical for the two task blocks, with the exception of the list lengths used. Note that embedded within each block were identical, matched six-word trials. Each task block in the behavioral study and each run of the short-list task in the fMRI study contained 10 trials of the critical six-word lists and 6 trials each of the other five list lengths, for a total of 40 trials in each block/run. Each run in the long-list task block during the fMRI study contained 5 trials of the critical six-word lists and 3 trials of each of the other five list lengths, for a total of 20 trials in each run. Within each block/run, trial lengths were pseudorandomly intermixed. A short (10-trial) practice block preceded each task in order to acquaint the participants with the tasks and encourage them to develop stable strategies.

Each list word remained on the screen for $500 \mathrm{msec}$, followed by a 500-msec interstimulus interval. Presenting the items in the list sequentially rather than simultaneously prevented the participants from knowing the total list length for a given trial until after the presentation of the final list item. The presentation of each word list was followed by a 3,000-msec retention interval. At the end of the retention interval, a probe word appeared for $1,500 \mathrm{msec}$, and the participants were given a maximum of 2,000 msec following the onset of the probe word to make a response. The next trial began immediately following the 2,000-msec response interval (behavioral study) or between $0 \mathrm{msec}$ and $5,000 \mathrm{msec}$ following the response interval (fMRI study). To enable event-related analyses, trials were jittered among $0 \mathrm{msec}, 2,500 \mathrm{msec}$, and 5,000 $\mathrm{msec}$, with an average intertrial interval of 2,500 msec (see Dale \& Buckner, 1997). Half of the probe words were chosen from words that appeared in the preceding word list (target trials), whereas the other half were words that had not been seen before (nontarget trials). For each list length, there was an equal number of target and nontarget trials. The participants in the behavioral study were not told that they would receive a later memory test for the nontarget probe words.

Surprise recognition test. At the end of the session, the participants in the behavioral study received a surprise yes/no recognition test. They were instructed to respond to each word that appeared on the computer screen by indicating whether or not they had seen the word at any point during the short-list or long-list task blocks. The participants were told that if they could not explicitly remember seeing the word, they should answer on the basis of their gut feeling as to whether or not the word seemed like one they had seen during the first two memory tasks.

The recognition task consisted of 160 words presented individually on the computer screen. Each word was chosen randomly from a pool of 80 old words and 80 new words. The words remained on the screen until the participants responded with a target buttonpress (if they felt they had seen the word during the first two task blocks), or a nontarget buttonpress (if they felt they had not seen the word during the first two task blocks). A 500-msec intertrial interval separated the recognition trials. The self-paced recognition task was designed to allow the participants to emphasize accuracy over speed. The recognition task was broken up into two blocks of trials, with 80 trials in each block. Each block of trials had an equal number of old and new items.

Scanning. Images were acquired on a 1.5-T Siemens Vision MRI scanner (Erlangen, Germany) with a standard circularly polarized head coil. A pillow and tape were used to minimize head movement, and headphones reduced scanner noise while allowing for communication with the participants. Both structural and functional images were acquired at each session. High-resolution $(1.25 \times 1 \times 1)$ structural images were acquired using a sagittal MP-RAGE 3-D T1weighted sequence (repetition time $[\mathrm{TR}]=9.7 \mathrm{msec}$, time to echo $[\mathrm{TE}]=4 \mathrm{msec}$, flip $=12^{\circ}$, inversion time $[\mathrm{TI}]=300 \mathrm{msec}$; Mugler $\&$ Brookeman, 1990). Functional images were acquired using an asymmetric spin-echo echo-planar sequence ( $\mathrm{TR}=2,500 \mathrm{msec}$, $\mathrm{TE}=37 \mathrm{msec}$, flip $=90^{\circ}$ ). Each image consisted of 18 contiguous, 7-mm-thick axial slices acquired parallel to the anterior-posterior commissure plane $(3.75 \times 3.75 \mathrm{~mm}$ in-plane resolution), allowing complete brain coverage at a high signal-to-noise ratio (Conturo et al., 1996). Visual stimuli were presented using PsyScope software (Cohen et al., 1993) running on an Apple PowerMac G4. An AmPro LCD projector (model 150) projected stimuli onto a screen positioned at the head of the bore. The participants viewed the stimuli on the screen through a mirror attached to the head coil. A fiber-optic light-sensitive keypress interfaced with the PsyScope Button Box was used to record the participants' performance.

\section{Data Analysis}

Behavioral data. Three sets of behavioral analyses were conducted to ensure that the participants were using quantitatively different strategies during the two blocks of the memory task. Each set of analyses included an analysis of the proportion of correct responses and the mean RTs to correct trials (with the exception of the recognition analysis, which is described below). One participant from the behavioral study was excluded from the behavioral analyses due to abnormally low-accuracy performance in the short-list block (more than $3 S D$ s below the mean at all list lengths), and 1 participant from the fMRI study was excluded from the behavioral and imaging analyses due to technical problems with the scanner. Initially, the analyses included the two between-participants factors of 
block order (short-list block first and long-list block first) and testing environment (inside and outside of the scanner). These factors did not interact with the factors of interest and, therefore, are not reported in the behavioral results.

In the first set of analyses, separate analyses of variance (ANOVAs) were conducted for the short- and long-list blocks because the nested design made a single ANOVA inappropriate. Although the experimental design appears factorial, with six levels of list length in each block, list length was nested within block. Therefore, a one-word increase in list length in the short-list block was not necessarily equivalent to a one-word increase in list length in the long-list block. The six-word trials were not included in these ANOVAs because they were analyzed separately in the second set of analyses using pairedsamples $t$ tests to compare performance on the six-word trials within each block. In the third analysis, the proportion of correctly recognized nontarget probe words from the short-list block was compared with that from long-list block. Because there were not enough trials to compare recognition performance only in the six-word trials (10 trials per cell), recognition performance was defined as the proportion of correctly identified words (hits) across all original list lengths in the short-list task block and in the long-list task block. That is, we performed the recognition analyses without using number of words in the list as an independent variable. Given the self-paced nature of the recognition test, we did not analyze the RT data. Alpha was set at .05 for all analyses.

Neuroimaging data. All functional images were first corrected for movement using a rigid-body rotation and translation correction (Friston, Williams, Howard, Frackowiak, \& Turner, 1996; Snyder, 1996) and registered to the participant's anatomical images (in order to correct for movement between the anatomical and functional scans). The data were then scaled to achieve a whole-brain mode signal value (used in place of a mean signal because of its reduced sensitivity to variation in brain margin definition) of 1,000 for each scanning run. This normalization procedure was conducted on a runby-run (rather than image-by-image) basis to reduce the effects of between-run scanner drift or instability without affecting within-run signal changes. The data were then spatially smoothed with an 8mm FWHM Gaussian kernel. The participants' structural images were transformed into standardized atlas space (Talairach \& Tournoux, 1988 ) using a 12-dimensional affine transformation (Woods, Cherry, \& Mazziotta, 1992; Woods, Grafton, Holmes, Cherry, \& Mazziotta, 1998). The functional images were then registered to the reference brain using alignment parameters derived for the structural scans.

A general linear model approach (Friston et al., 1996) was used to estimate parameter values for the various time points within the hemodynamic response epoch. Because memory trials were up to $16 \mathrm{sec}$ in duration (11-word trials), the hemodynamic response epoch was taken to be $35 \mathrm{sec}$ (14 scanning frames), and in order to avoid having a singular design matrix, the time courses for each participant were constrained such that they had a zero mean. The event-related estimates for the time-course data were then submitted to a group analysis using voxel-wise random-effects model ANOVAs. Eventrelated responses can be determined in this approach by using time (i.e., frame) as a factor of interest and examining significant effects of this factor (main effect and interactions). The primary advantage of this approach is that it makes no a priori assumptions about the particular shape of the hemodynamic response (Buckner \& Braver, 1999). Given the complex nature of trials (i.e., that they were composed of multiple events), the hemodynamic response for a trial was also likely to be complex in shape and difficult to estimate from established models. We conducted random-effects voxel-wise ANOVAs with time as the single within-participants factor (14 levels corresponding to each frame in the trial). As in the behavioral analysis, separate ANOVAs were conducted for the short-list and long-list blocks. Furthermore, the number-of-words variable was not included in these analyses, because the factors of time (frame) and number of words were highly correlated (increasing the number of words in the trial also increased the number of frames in the trial), which violates one of the assumptions of the ANOVA (Hays, 1994).

Regions showing a reliable change in activity during the course of the trial in either block were identified in the main effect of time, collapsed across list lengths but excluding the six-word trials. Note that the exclusion of the six-word trials ensures that identified regions showed sensitivity to the task in general. The main effect of time was thresholded at $z>3.00$ with contiguous clusters of 70 or more voxels, maintaining an image-wise false positive rate of $p<.05$ (Ollinger \& McAvoy, 2000). The remaining voxels were then segregated into 10-mm spherical regions of interest (ROIs) with peaks at least $10 \mathrm{~mm}$ apart. Any ROIs that had a negative average level of activation across all frames within a task block were excluded from further analyses. Activation within ROIs was averaged across voxels before the ROI-based ANOVAs were conducted, and the latter were corrected for multiple comparisons in order to maintain family-wise error rates of $p=.05$. ROIs that showed a main effect of time in both the short-list and long-list blocks were subjected to six-word analyses.
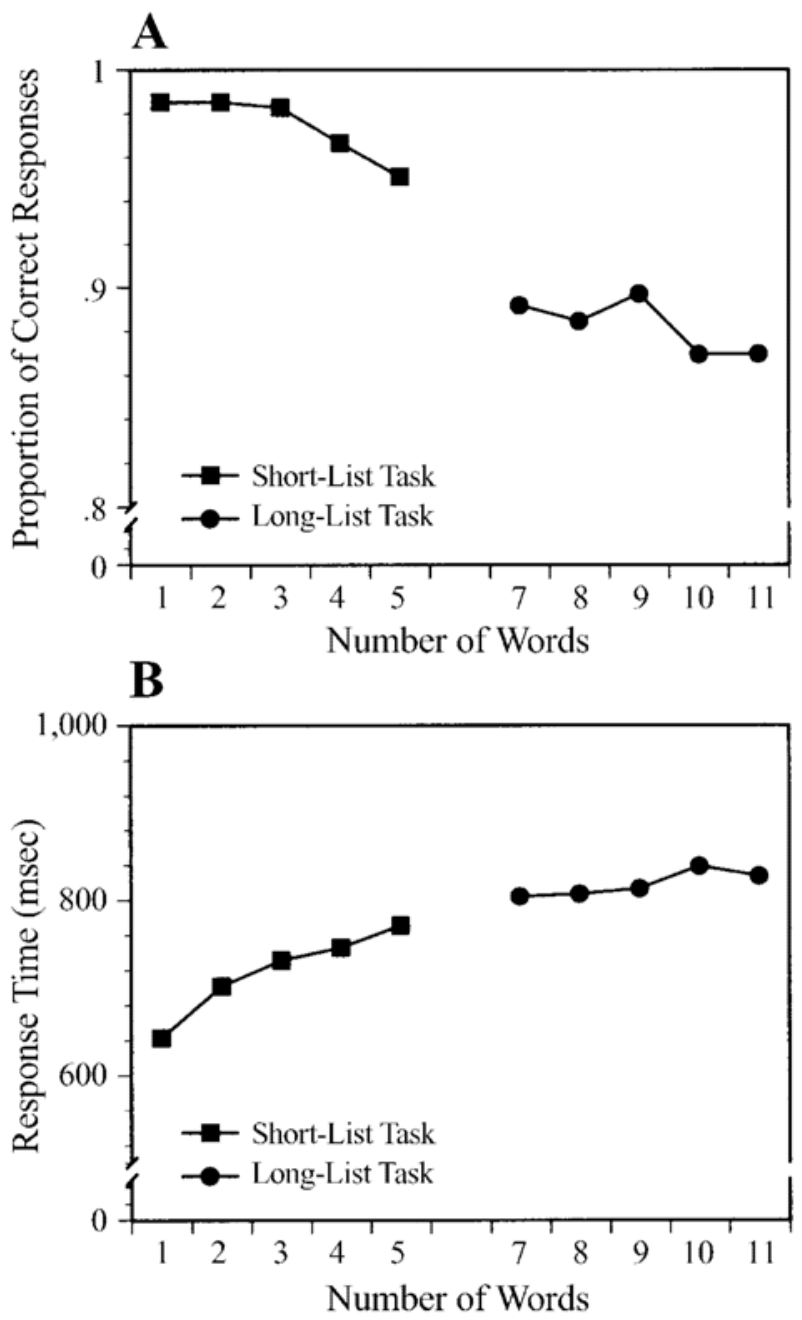

Figure 2. Different patterns of behavioral performance on the short-list and long-list task blocks provide evidence that the participants were biased toward qualitatively different memory strategies. The effects of list length on (A) accuracy and (B) response time (RT) are consistent with the use of maintenancefocused and retrieval-focused strategies in the short-list and longlist task blocks, respectively. 


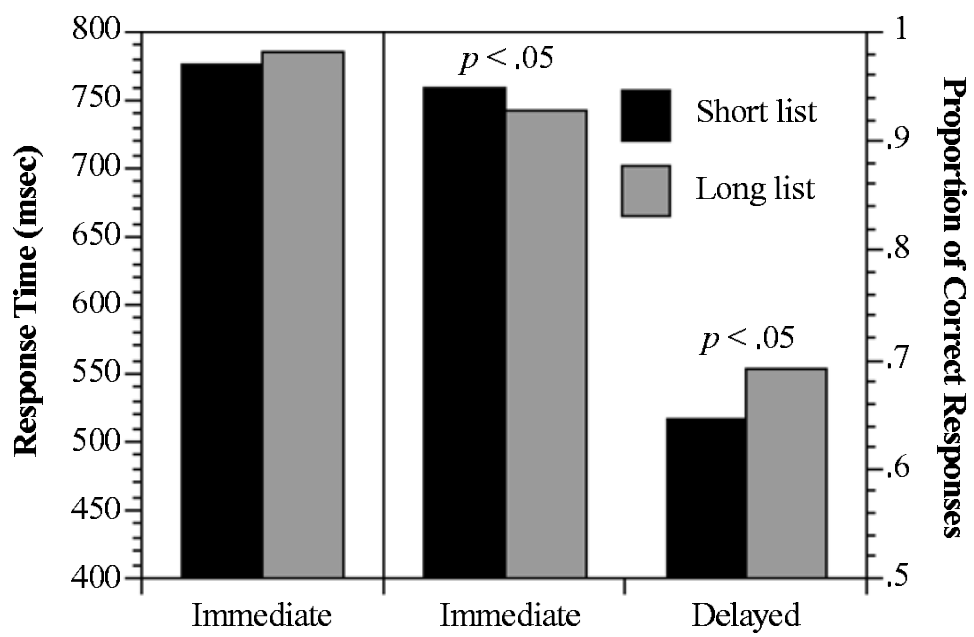

Type of Recognition

Figure 3. Mean response times (RTs) and proportion of correct responses for the six-word trials appearing in the context of the short-list and long-list task blocks (immediate recognition) are shown with the mean proportion of correctly recognized nontarget probe words in the delayed recognition task (delayed recognition), averaged across all list lengths. The more accurate immediate recognition responses in the short-list task block contrast with the more accurate delayed recognition responses in the long-list task block.

In the six-word trial analyses, a comparison was made of the time courses of activation in the short-list and long-list task blocks within ROIs to identify regions showing strategy-dependent change. These regions were defined as those showing task-related activation in the short-list and long-list blocks as well as in the six-word trials, with time courses of activation in the six-word trials that differed depending on the block in which they appeared (i.e., those that showed a significant interaction of task and time). If a region showed a significant effect of block type (short vs. long list) but also showed an interaction of this effect with task order, an additional control analysis was performed. The control analysis was a 2 (block) $\times 2$ (time) between-participants ANOVA using only the first block that the participants had performed to determine whether the effect of strategydependent change was present independently of the effects of task order. Only analyses of the regions meeting these criteria for strategydependent change are reported below.

Regions showing strategy-depende nt change were further analyzed to characterize the effects of strategy on the time course of brain activation. Activation over Frames 1 through 4 was averaged (first half of trial) and compared with the average activation over Frames 5 through 8 in a series of $2 \times 2$ ANOVAs, with trial half (first four frames vs. last four frames) and task (short-list block vs. long-list block) as the two within-participants variables. Because these regions had already survived our conservative selection criteria, showing a significant interaction of task and time in the six-word trials as well as a main effect of time in the six-word trials, the short-list task block, and the long-list task block (with corrections made for multiple comparisons in each analysis), we adopted a relatively liberal threshold, with alpha $\leq .05$, uncorrected, for these tests.

\section{RESULTS}

\section{Behavioral Results}

Short-list and long-list block effects. As list length increased, the proportion of correct responses in the shortlist block decreased $[F(4,248)=6.86, p<.001]$, but that in the long-list block did not $[F(4,248)=1.24$, n.s.; see
Figure 2A]. In addition, increases in list length led to an increase in RTs in the short-list and long-list blocks $[F(4,248)=55.26, p<.001$, and $\mathrm{F}(4,248)=4.07, p<.005$, respectively; see Figure $2 \mathrm{~B}]$. However, follow-up pairwise comparisons indicated that RTs increased consistently only in the short-list block: In the long-list block, there was a significant increase in RTs only between the 9- and 10-word lists.

Six-word trial analyses. As is shown in Figure 3, the proportion of accurate responses to six-word trials appearing in the context of the short-list block $(M=.95$, $S D=.06$ ) was significantly greater than the proportion of accurate responses to six-word trials appearing in the context of the long-list block $[M=.93, S D=.07, t(62)=$ $1.46, p<.02]$. Although there was a trend for mean RTs to correct six-word trials to be shorter in the context of the short-list block $(M=775.48 \mathrm{msec}, S D=151.02)$ than in the context of the long-list block $(M=784.60 \mathrm{msec}, S D=$ $149.60)$, this difference was not statistically significant $[t(62)=-0.91$, n.s. $]$.

Recognition analyses. There was a significantly higher proportion of correctly identified nontarget probe words from the long-list task $(M=.69, S D=.15)$ than from the short-list task $[M=.65, S D=.18, t(38)=2.64, p=.01$; see Figure 3]. The false alarm rate was .38. Note that we report a single false alarm rate because the nontarget probe words from the short- and long-list task blocks were intermixed in the surprise recognition test.

\section{Neuroimaging Results}

Short-list and long-list block effects. Figure 4 shows task-related activity in the short-list and long-list task blocks overlaid onto the cortical surface, and Table 1 shows 


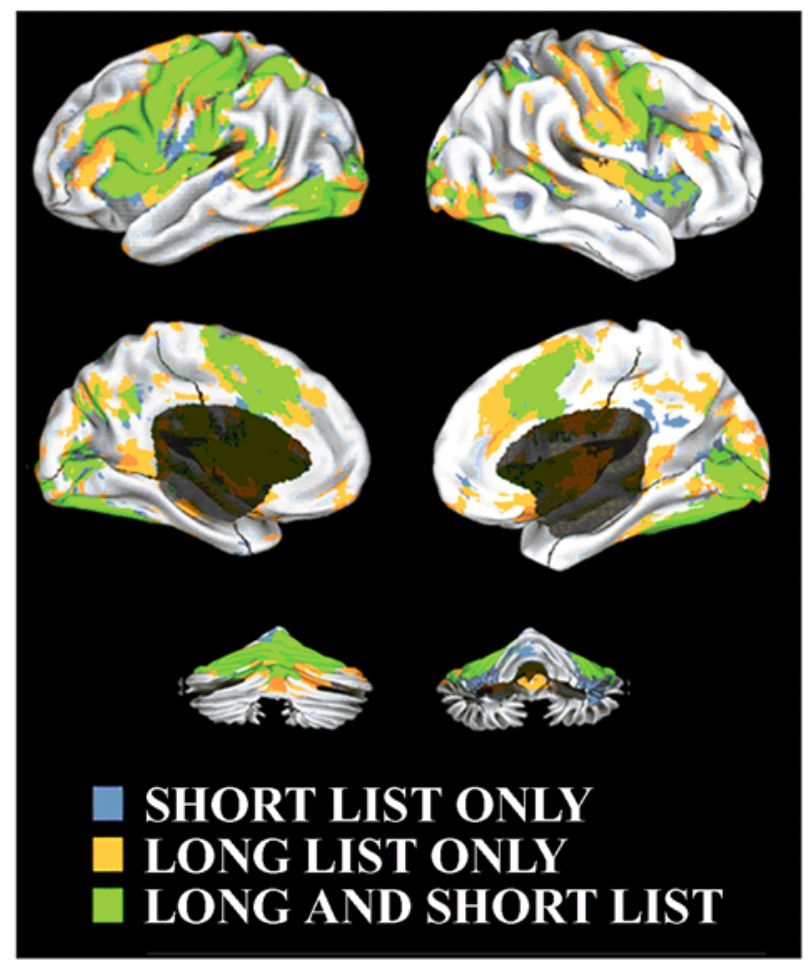

Figure 4. Activation maps showing the extent of modulation by the short- and long-list tasks. Regions showing evoked responses to only the short-list task are indicated in blue, and regions showing evoked responses to only the long-list task are indicated in orange. The majority of the activated regions showed task-related activation to both the short- and long-list task blocks (these regions of overlap are shown in green).

the locations of these ROIs. These regions were identified by a main effect of time in either the short- or the long-list task block. As is indicated in the figure, task-sensitive activation was found in a large number of regions across the brain, and there was a large degree of overlap in regions activated during the short- and long-list task blocks. Overall, $84 \%$ of the regions identified showed activation in both task blocks. It is difficult to draw conclusions regarding the regions that show a significant evoked response in only one task block, since the lack of significant activity in the other task block may reflect insufficient power to detect a true response rather than actual selectivity of function.

Six-word trial analyses. Of the regions that showed significant increases in activation in response to both the short-list and long-list blocks, 13 showed significant interactions of block (short-list and long-list) and time in the critical six-word trials $(p<.02$, corrected for multiple comparisons), indicating that these regions showed strategydependent changes in activation (see Table 2).

Seven of the strategy-sensitive regions were found within frontal cortex, in posterior motor areas (e.g., Brodmann's areas 4 and 6), as well as in posterior inferior and midlateral PFC (areas 44, 45, 47, and 9). The remaining strategy-sensitive brain regions were found in the occipital cortex, the temporal cortex, and the cerebellum. These regions are shown overlaid onto the cortical surface in Figure 5, which also displays the time courses of activation across the six-word trials for selected regions. The most common pattern of activation dynamics observed across the regions showing strategy-dependent change was one in which activation was greater during the long-list task block at the start of the trial (presumably during frames when the participants were encoding the list of words) and/or during the short-list task block at the end of the trial (presumably when the participants were preparing and making a response).

The results of the within-trial ANOVAs indicated that nine of the strategy-dependent change regions showed a significant crossover interaction (see Table 2), with greater activation at the start of the trial relative to the end of the trial in the long-list task, and greater activation at the end of the trial relative to the start of the trial in the short-list task (see Figure 5). In contrast to these patterns, the left anterior frontal region showed a significant interaction in the opposite direction, and the right frontal operculum showed greater activation in the short-list block than in the long-list block during the middle and latter parts of the trial. Two of the cerebellar regions $(0,-78,-21$, and 45 , $-68,-29)$ showed greater activation during the long-list block than during the short-list block throughout the trial.

\section{DISCUSSION}

In this study, we observed strategy-dependent changes in behavior and brain activation independent of explicit task differences. Critically, the participants engaged these different strategies even on trials that were explicitly matched on memory load, retention interval, and encoding and retrieval duration. These strategies were first differentiated behaviorally by a distinct pattern of task performance and were subsequently differentiated anatomically by unique time courses of neural activation within specific brain regions activated by both blocks of the memory task.

The behavioral performance data provided three separate indications that different mnemonic strategies were being biased across the short- and long-list task blocks. First, accuracy and RTs were consistently related to list length in the short-list task block but not in the long-list task block. Previous investigators have taken this pattern as prima facie evidence of a qualitative disjunction between STM and LTM processes occurring around the boundary of STM capacity (e.g., Burrows \& Okada, 1975; Sternberg, 1966, 1967). Second, on matched six-word trials, the participants were more accurate when those trials were presented in the context of the short-list task block. This novel finding provides strong behavioral evidence that the participants engaged different mnemonic processes during the short-list and long-list task blocks. Third, on a surprise delayed recognition test, the participants showed better memory for nontarget probe words presented in the context of the long-list task block. Such a pattern, which would otherwise be counterintuitivegiven that three times 
Table 1

Regions Activated in the Short- and Long-List Task Blocks

\begin{tabular}{|c|c|c|c|c|c|c|c|}
\hline \multirow[b]{2}{*}{ Region } & \multicolumn{3}{|c|}{ Coordinates } & \multirow[b]{2}{*}{ Hem } & \multirow[b]{2}{*}{ BA } & \multirow[b]{2}{*}{ Peak $Z$} & \multirow[b]{2}{*}{ \# Voxels } \\
\hline & $x$ & $y$ & $z$ & & & & \\
\hline \multirow[t]{10}{*}{ Frontal } & -44 & 29 & 7 & $\mathrm{~L}$ & $45 / 46$ & 7.71 & 143 \\
\hline & -42 & -15 & 57 & $\mathrm{~L}$ & 4 & 13.74 & 86 \\
\hline & -4 & 50 & -11 & $L$ & $10 / 11$ & 5.37 & 69 \\
\hline & 5 & 50 & -9 & $R$ & $10 / 11$ & 5.69 & 57 \\
\hline & 41 & -11 & 59 & $\mathrm{R}$ & $4 / 6$ & 8.69 & 94 \\
\hline & 44 & -4 & 40 & $\mathrm{R}$ & 6 & 10.95 & 124 \\
\hline & 45 & 30 & 30 & $\mathrm{R}$ & $9 / 46$ & 8.31 & 127 \\
\hline & 48 & -8 & 52 & $\mathrm{R}$ & 4 & 11.98 & 62 \\
\hline & 50 & -6 & 47 & $\mathrm{R}$ & 4 & 12.08 & 64 \\
\hline & 61 & -10 & 29 & $\mathrm{R}$ & 4 & 6.62 & 130 \\
\hline \multirow[t]{4}{*}{ Limbic } & 1 & -24 & 29 & $R$ & 23 & 5.16 & 124 \\
\hline & 4 & -47 & 7 & $R$ & $29 / 30$ & 5.15 & 128 \\
\hline & 5 & 24 & 31 & $R$ & 32 & 5.88 & 127 \\
\hline & 10 & 44 & -4 & $\mathrm{R}$ & 32 & 5.16 & 67 \\
\hline \multirow[t]{6}{*}{ Occipital } & -27 & -87 & -9 & $\mathrm{~L}$ & 18 & 16.26 & 132 \\
\hline & -19 & -93 & -7 & $\mathrm{~L}$ & 17 & 15.48 & 129 \\
\hline & -7 & -74 & 33 & $\mathrm{~L}$ & 19 & 7.47 & 132 \\
\hline & 3 & -76 & 31 & $\mathrm{R}$ & 19 & 6.94 & 126 \\
\hline & 20 & -91 & -5 & $\mathrm{R}$ & 18 & 13.86 & 154 \\
\hline & 36 & -83 & -16 & $\mathrm{R}$ & $18 / 19$ & 9.96 & 141 \\
\hline \multirow[t]{8}{*}{ Parietal } & -42 & -28 & 58 & $\mathrm{~L}$ & 40 & 15.70 & 91 \\
\hline & -30 & -57 & 41 & $\mathrm{~L}$ & 40 & 11.62 & 128 \\
\hline & -3 & -57 & 66 & $\mathrm{~L}$ & 7 & 5.28 & 93 \\
\hline & 0 & -53 & 36 & & 31 & 5.11 & 134 \\
\hline & 27 & -53 & 41 & $\mathrm{R}$ & 7 & 9.61 & 155 \\
\hline & 38 & -34 & 63 & $R$ & 40 & 5.69 & 73 \\
\hline & 50 & -28 & 24 & $\mathrm{R}$ & 40 & 4.72 & 132 \\
\hline & 58 & -22 & 43 & $\mathrm{R}$ & 2 & 4.97 & 115 \\
\hline \multirow[t]{7}{*}{ Sublobar } & -14 & -18 & 14 & $\mathrm{~L}$ & thal & 7.93 & 127 \\
\hline & -12 & 6 & -7 & $L$ & $b g$ & 7.46 & 89 \\
\hline & 9 & -22 & 3 & $\mathrm{R}$ & thal & 6.75 & 147 \\
\hline & 9 & 9 & -7 & $\mathrm{R}$ & bg & 6.94 & 137 \\
\hline & 14 & -15 & 18 & $\mathrm{R}$ & thal & 6.87 & 143 \\
\hline & 23 & -18 & -6 & $\mathrm{R}$ & bg & 5.00 & 105 \\
\hline & 34 & -13 & 3 & $\mathrm{R}$ & bg & 6.22 & 132 \\
\hline \multirow[t]{5}{*}{ Temporal } & -45 & -44 & 21 & $\mathrm{~L}$ & 22 & 5.24 & 139 \\
\hline & -34 & 12 & -30 & $L$ & 38 & 5.60 & 85 \\
\hline & 12 & -39 & 3 & $R$ & 30 & 5.19 & 108 \\
\hline & 48 & -46 & 9 & $\mathrm{R}$ & $21 / 22$ & 5.90 & 126 \\
\hline & 50 & -23 & 13 & $\mathrm{R}$ & 42 & 4.89 & 119 \\
\hline \multirow[t]{4}{*}{ Other } & -33 & -29 & -27 & $\mathrm{~L}$ & $\mathrm{cb}$ & 5.41 & 94 \\
\hline & -8 & -26 & 0 & $\mathrm{~L}$ & $\mathrm{mb}$ & 7.99 & 115 \\
\hline & 5 & -58 & -33 & $R$ & $c b$ & 4.80 & 126 \\
\hline & 37 & -71 & -15 & $\mathrm{R}$ & $\mathrm{cb}$ & 11.22 & 127 \\
\hline
\end{tabular}

Note-Regions in italics showed activation specific to one task. All other regions showed increases in activation in response to the short- and long-list task blocks. $x, y, z$, coordinates of ROI; Hem, hemisphere; BA, Brodmann's area; Peak Z, Peak $Z$ statistic in ROI; \# Voxels, number of voxels in ROI; thal, thalamus; bg, basal ganglia; cb, cerebellum; mb, midbrain.

as many items were presented in the long-list task block, indicates that the participants were preferentially processing probe items during long-list trials. Taken together, these performance patterns are consistent with the interpretation that in the short-list task block the participants preferentially engaged maintenance-focused processes, whereas in the long-list task block they preferentially engaged retrieval-focused processes.

The analysis of the neuroimaging data focused on the six-word trials that were identically matched on all explicit task requirements. The focus on identically matched trials contrasts with that of previous studies investigating differences in the neural substrates of STM and LTM (Braver et al., 2001; Cabeza et al., 2002; Ranganath et al., 2003), which have not explicitly matched task requirements. We identified 10 cortical regions and 3 cerebellar regions that showed evidence of strategy-dependent change (see Figure 5 and Table 2). As we had predicted, the frontal cortex had the largest number of regions showing strategy-dependent change. Some of these regions were located within structures that are oriented primarily toward response selection (e.g., frontal operculum) and execution (e.g., primary motor cortex), whereas others were within lateral $\mathrm{PFC}$ regions that have been implicated 
Table 2

Locations, Peak Z Statistics, and Number of Voxels

in Regions Showing Strategy-Dependent Change

\begin{tabular}{lrrrcccc}
\hline & \multicolumn{3}{c}{ Coordinates } & & & & \\
\cline { 2 - 4 } Region & $x$ & $y$ & $z$ & Hem & BA & Peak $Z$ & \# Voxels \\
\hline Frontal & -48 & -9 & 43 & $\mathrm{~L}$ & $4 / 6$ & 14.18 & $128^{*}$ \\
& -42 & 10 & 26 & $\mathrm{~L}$ & $44 / 9$ & 11.15 & $154^{*}$ \\
& -40 & -3 & 33 & $\mathrm{~L}$ & $44 / 6$ & 10.25 & $151^{*}$ \\
& -28 & 43 & -10 & $\mathrm{~L}$ & $10 / 11$ & 4.70 & $50^{*}$ \\
& -4 & -1 & 58 & $\mathrm{~L}$ & 6 & 13.03 & $105^{*}$ \\
& 33 & 17 & 11 & $\mathrm{R}$ & Operculum & 9.02 & 152 \\
Occipital & 51 & 16 & -1 & $\mathrm{R}$ & 47 & 6.55 & $100^{*}$ \\
& -40 & -70 & -10 & $\mathrm{~L}$ & $19 / 37$ & 14.21 & $155^{*}$ \\
Temporal & 17 & -88 & -9 & $\mathrm{R}$ & 18 & 11.06 & $77^{*}$ \\
Cerebellum & -53 & -49 & 4 & $\mathrm{~L}$ & $21 / 22$ & 10.75 & $157^{*}$ \\
& 0 & -78 & -21 & & & 7.99 & 133 \\
& 17 & -83 & -16 & $\mathrm{R}$ & & 10.43 & $87^{*}$ \\
& 45 & -68 & -29 & $\mathrm{R}$ & & 7.63 & 144 \\
\hline
\end{tabular}

Note-All regions showed increases in activation in response to the short- and long-list task blocks. $x, y, z$, coordinates of ROI; Hem, hemisphere; BA, Brodmann's area; Peak Z, Peak Z statistic in ROI; \# Voxels, number of voxels in ROI. *Predicted pattern of activation.

in controlled verbal processing and working memory (Cabeza \& Nyberg, 2000; D’Esposito et al., 1998; Gabrieli, Poldrack, \& Desmond, 1998; Smith \& Jonides, 1999; Thompson-Schill, D’Esposito, Aguirre, \& Farah, 1997). For example, we found activity in a left-lateralized posterior frontal region associated with phonological or lexical processing (area 44/6; Poldrack et al., 1999; Smith \& Jonides, 1997; Wagner, 1999; Wagner, Poldrack, et al., 1998), in supplementary motor regions that have been associated with articulatory rehearsal (Chein \& Fiez, 2001; Fiez et al., 1996; Smith \& Jonides, 1998), and in a midlateral PFC (area 44/9) region widely thought to be associated with maintenance and manipulation of information within working memory (Braver et al., 1997; Cohen et al., 1997; D’Esposito et al., 1998; Smith \& Jonides, 1999). Together, the network of identified frontal regions comprises brain areas that previous research has associated with response selection as well as with aspects of verbal processing, memory encoding, and retrieval. It is therefore not surprising that these regions would be sensitive to the particular processing strategy used to perform the verbal memory task utilized in this study.

The specific pattern of activity dynamics exhibited by these brain regions in the different task contexts provides insight into how strategy effects modulate processing. In particular, the majority of identified regions showed greater activation for the long-list task block in comparison with the short-list task block during the beginning of the trial, but then reversed to show greater activity during the shortlist task block in comparison with the long-list task block toward the end of the trial. This pattern of crossover dynamics found in the time courses of activation in most of the strategy-sensitive brain regions is the critical finding in these results. Each region was activated to some extent during task performance regardless of task context or memory strategy, but the fact that the activity dynamics in most regions showed a characteristic strategy-dependent pattern indicates that the processing functions subserved by these regions may be differentially recruited during STM and LTM tasks.
When an STM strategy was biased (i.e., in the short-list task block), these regions showed increased activation later on in the trial, presumably during the delay and response periods. These activation increases may have reflected the active articulatory rehearsal of verbal information during the delay and the direction of target detection processes toward the onset of the probe stimulus. The lateral posterior PFC regions in particular showed a pattern of activation that is consistent with a role in articulatory processing. These regions increased in activation during the latter half of the trial during the short-list task, when the participants reported subvocally rehearsing the words in the list, and during the initial period of the trial during the long-list task, when many participants reported elaborating on the sound of the words in the list.

Conversely, when an LTM strategy was biased (i.e., in the long-list task block), most regions showed an early increase in activation, presumably during the encoding period, followed by decreasing or sustained activation over the course of the trial. This early activation increase may have reflected processes associated with enhanced or elaborative encoding of the to-be-remembered items, with decreased rehearsal and anticipation of the target during the latter part of the trial. Interestingly, in a left anterior PFC region (area 10/11), we observed greater activity in the long-list block at the end of the trial. This region is near (though somewhat inferior to) the location of brain regions typically activated in tasks requiring episodic retrieval from LTM (Lepage et al., 2000). This retrievalrelated anterior PFC activity has typically been observed in the right hemisphere; however, more recent studies have shown that left anterior PFC activity is related to retrieval success (Konishi, Wheeler, Donaldson, \& Buckner, 2000; McDermott, Jones, Petersen, Lageman, \& Roediger, 2000). Given the established role of the anterior PFC in LTM retrieval, it is possible that the activity of this region may reflect a shift in mnemonic strategies toward episodic retrieval in the long-list task block.

These patterns of strategy-modulated brain activation are consistent with the behavioral results. In the short-list 

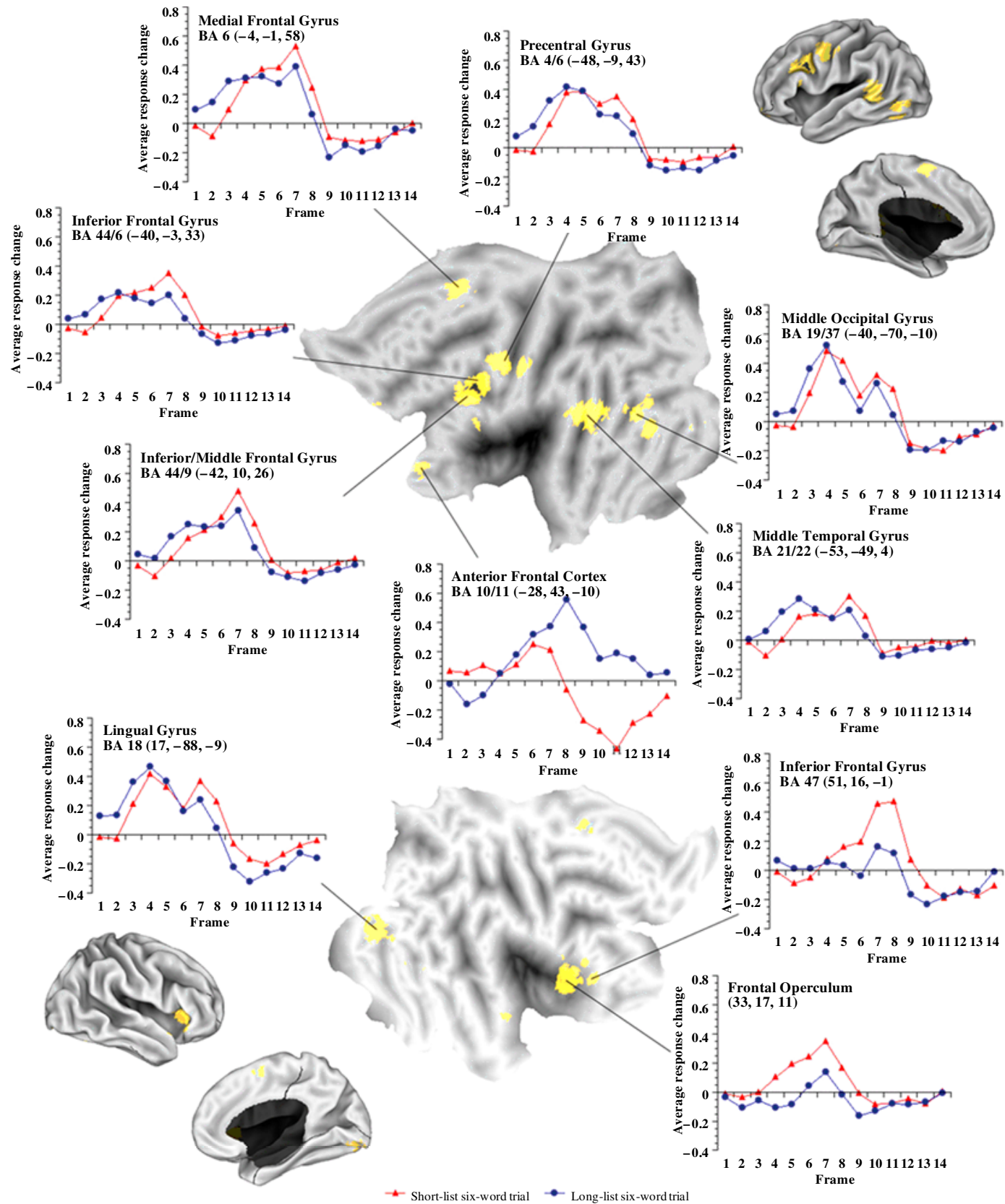

Figure 5. Time courses of activation for 10 regions showing evidence of strategy-dependent change, as well as surface-based maps of the cortical surface indicating the locations of regions showing strategy-dependent change (these maps were produced using CARET; Van Essen, 2002a, 2002b; Van Essen et al., 2001; Van Essen, Drury, Harwell, \& Hanlon, 2002). The blue lines correspond to the long-list task, and the red lines correspond to the short-list task.

condition, the facilitation in immediate task performance may have been due to the increased activity during the delay and response period of brain regions supporting anticipatory active maintenance and articulatory rehearsal. In contrast, in the long-list condition, the facilitation in delayed recognition may have been due to increased activity in brain regions involved in helping to solidify the episodic trace of probe items.
These results make an important contribution to the memory literature by highlighting the fact that the mnemonic processes engaged by specific brain regions are flexible and open to influences of the task environment. Most likely, there are additional brain regions that show effects of strategy-dependent change that were not robust enough to be detected in the present study (e.g., the brain regions showing general task-related activation in the short- and 
long-list blocks). Visual inspection of the time courses of regions showing task-related activation in the short-list and long-list task blocks (shown in Figure 4 and Table 1) hinted at the presence of weaker strategy effects, lending support to this hypothesis. Consequently, a more powerful design might reveal significant strategy-dependentchanges in additional brain regions beyond those identified here.

The regions that did show reliable strategy-dependent change in the present study appear to be a subset of regions typically activated during tasks requiring maintenancefocused or retrieval-focused processing (Buckner, 1996; Buckner et al., 1999; Buckner \& Koutstaal, 1998; D'Esposito et al., 1998; Rypma, Prabhakaran, Desmond, Glover, \& Gabrieli, 1999; Smith \& Jonides, 1997), including regions found in recent studies in which performance on STM and LTM tasks has been directly compared within individual participants (Braver et al., 2001; Cabeza et al., 2002; Ranganath et al., 2003). These results extend previous findings by using implicit context to bias mnemonic processing, leading to a greater separation of STM and LTM processes from STM and LTM tasks. This experimental strategy has been strongly advocated by memory researchers working within the tradition of cognitive psychology (e.g., Jacoby, 1991). Although our design most likely did not eliminate LTM processes from the short-list (STM) task or vice versa, our results direct attention to the benefits of this biasing approach for neuroimaging research, while highlighting the strategic nature of mnemonic processing and the brain activity that supports it.

One other important feature of these results is that we observed very few regions showing task-specific activation. That is, the majority of the regions that showed taskrelated activation to the short-list task block also showed task-related activation to the long-list task block and vice versa. We take this large degree of overlapping activation as evidence that STM and LTM tasks by themselves do not isolate STM and LTM processes and may even rely on common neural substrates. It could be argued that the long-list task block was more similar to an STM task than to an LTM task, and it was this similarity, rather than a reliance on common neural substrates, that led to the large degree of overlapping activation. However, given the strong behavioral evidence that the participants were using quantitatively different mnemonic processes to perform the two task blocks, along with recent evidence that STM and LTM tasks less stringently matched on explicit task demands also show a large degree of overlapping activation across tasks (Ranganath et al., 2003), we believe our results support theories that STM and LTM processes rely primarily on common neural substrates (see, e.g., Cowan, 1999).

We hope that future research will capitalize on the type of experimental design we have utilized in this study. We have shown that participants' preferred memory strategies can be biased while explicit task demands are held constant. Moreover, the success of our manipulation shows that such effects can be produced via subtle contextual changes of the task environment, independent of explicit changes in task demands. Future studies might explore other methods for biasing task strategy that do not involve manipulations of memory load. Indeed, different types of strategy manipulations might be more powerful for detecting effects of strategy modulation, since one limitation of our design is that the explicitly matched trials that were the focus of our investigation constituted only $25 \%$ of the trials in the study. This limitation is easily overcome: For example, we would predict that participants would be biased to use a retrieval-focused strategy when maintenancefocused processes are prevented, such as when distracting information or articulatory suppression occurs between the encoding and retrieval periods of the trial. This type of manipulation would allow all trials to be matched on memory load, retention interval, and encoding and retrieval duration while significantly increasing the power to detect strategy-dependent changes in brain activation. The present method may also be used in future studies to explore memory deficits in patient populations, such as in patients with amnesia or frontal lobe damage. In these populations, we would predict that performance on the six-word trials would be dramatically impaired when the patients were biased to use a deficient mnemonic strategy (e.g., a maintenance-focused strategy in frontal lobe patients and a retrieval-focused strategy in amnesic patients) to perform the memory task, but their performance on the six-word trials would be relatively normal when they were biased to use an intact memory strategy.

\section{REFERENCES}

AtKinson, R. C., \& Shiffrin, R. M. (1971). The control of short-term memory. Scientific American, 225(2), 82-90.

Barch, D. M., Braver, T. S., Nystrom, L., Forman, S. D., Noll, D. C., \& Cohen, J. D. (1997). Dissociating working memory from task difficulty in human prefrontal cortex. Neuropsychologia, 35, 1373-1380.

Braver, T. S., Barch, D. M., Kelley, W. M., Buckner, R. L., Cohen, N. J., Miezin, F. M., Snyder, A. Z, Ollinger, J. M., Akbudak, E., Conturo, T. E., \& Petersen, S. E. (2001). Direct comparison of prefrontal cortex regions engaged by working and long-term memory tasks. NeuroImage, 14, 48-59.

Braver, T. S., Cohen, J. D., Nystrom, L. E., Jonides, J., Smith, E. E., \& Noll, D. C. (1997). A parametric study of prefrontal cortex involvement in human working memory. Neurolmage, 5, 46-62.

Brewer, J. B., Zhao, Z., Glover, G. H., \& Gabrieli, J. D. E. (1998). Making memories: Brain activity that predicts how well visual experience will be remembered. Science, 281, 1185-1187.

Buckner, R. L. (1996). Beyond HERA: Contributions of specif ic prefrontal brain areas to long-term memory retrieval. Psychonomic Bulletin \& Review, 3, 149-158.

BUCKNER, R. L., \& BRAVER, T. S. (1999). Event-related functional MRI. In P. Bandettini \& C. Moonen (Eds.), Functional MRI (pp. 441-450). New York: Springer-Verlag.

Buckner, R. L., Kelley, W. M., \& Petersen, S. E. (1999). Frontal cortex contributes to human memory formation. Nature Neuroscience, 2, 1-4.

Buckner, R. L., \& KoutstaAl, W. (1998). Functional neuroimaging studies of encoding, priming, and explicit memory retrieval. Proceedings of the National Academy of Sciences, 95, 891-898.

Burrows, D., \& OKADA, R. (1975). Memory retrieval from long and short lists. Science, 188, 1031-1033.

Cabeza, R, Dolcos, F., Graham, R, \& Nyberg, L. (2002). Similarities and differences in the neural correlates of episodic memory retrieval and working memory. NeuroImage, 16, 317-330.

CabezA, R. \& Nyberg, L. (2000). Imaging cognition II: An empirical 
review of 275 PET and fMRI studies. Journal of Cognitive Neuroscience, 12, 1-47.

CABeza, R. \& Nyberg, L. (2002). Seeing the forest through the trees: The cross-function approach to imaging cognition. In A. Zani \& A. M. Proverbio (Eds.), The cognitive electrophysiology of mind and brain (pp. 41-65). San Diego: Academic Press.

Capitani, E., Della Salla, S., Logie, R. H., \& Spinnler, H. (1992). Recency, primacy, and memory: Reappraising and standardising the serial position curve. Cortex, 28, 315-342.

Carlesimo, G. A., Sabbadini, M., Fadda, L., \& Caltagirone, C. (1995). Different components in word-list forgetting of pure amnesics, degenerative demented and healthy subjects. Cortex, 31, 735-745.

CheIn, J. M., \& FiEZ, J. A. (2001). Dissociation of verbal working memory system components using a delayed serial recall task. Cerebral Cortex, 11, 1003-1014.

Cohen, J. D., MacWhinney, B., Flatt, M., \& Provost, J. (1993). PsyScope: An interactive graphic system for designing and controlling experiments in the psychology laboratory using Macintosh computers. Behavior Research Methods, Instruments, \& Computers, 25, 257-271.

Cohen, J. D., Perlstein, W. M., Braver, T. S., Nystrom, L. E., Noll, D. C., Jonides, J., \& SMITH, E. E. (1997). Temporal dynamics of brain activation during a working memory task. Nature, 386, 604-608.

Conturo, T. E., McKinstry, R. C., Akbudak, E., Snyder, A. Z., YANG, T., \& RAICHLE, M. E. (1996). Sensitivity optimization and experimental design in functional magnetic resonance imaging. Society for Neuroscience Abstracts, 22, 7.

Courtney, S. M., Ungerleider,L. G., KeIl, K., \& Haxby, J. V. (1997). Transient and sustained activity in a distributed neural system for human working memory. Nature, 386, 608-612.

Cowan, N. (1999). An embedded process model of working memory. In A. Miyake \& P. Shah (Eds.), Models of working memory: Mechanisms of active maintenance and executive control (pp.62-101). Cambridge: Cambridge University Press.

Crowder, R. G. (1969). Behavioral strategies in immediate memory. Journal of Verbal Learning \& Verbal Behavior, 8, 524-528.

DALE, A. M., \& Buckner, R. L. (1997). Selective averaging of rapidly presented individual trials using fMRI. Human Brain Mapping, 5, 329-340.

D'Esposito, M., Aguirre, G. K., Zarahn, E., Ballard, D., Shin, R. K., \& LEASE, J. (1998). Functional MRI studies of spatial and nonspatial working memory. Cognitive Brain Research, 7, 1-13.

Eldridge,L. L., Knowlton, B. J., Furmanski, C. S., Bookheimer,S. Y., \& ENGEL, S. A. (2000). Remembering episodes: A selective role for the hippocampus during retrieval. Nature Neuroscience, 3, 1149-1152.

Engle, R. W., \& ORAnSKY, N. (1999). The evolution from short-term memory to working memory: Multi-store to dynamic models of temporary storage. In R. J. Sternberg (Ed.), The concept of cognition (pp. 515-555). Cambridge, MA: MIT Press.

Fiez, J. A., Raife, E. A., Balota, D. A., Schwarz, J. P., Raichle, M. E., \& Petersen, S. E. (1996). A positron emission tomography study of the short-term maintenance of verbal information. Journal of Neuroscience, 16, 808-822.

Friston, K. J., Williams, S., Howard, R, Frackowiak, R. S. J., \& TURNER, R. (1996). Movement-related effects in fMRI time-series. Magnetic Resonance in Medicine, 35, 346-355.

FusteR, J. M. (1989). The prefrontal cortex: Anatomy, physiology, and neuropsychology of the frontal lobe. New York: Raven.

Gabrieli, J. D. E., Poldrack, R. A., \& Desmond, J. E. (1998). The role of left prefrontal cortex in language and memory. Proceedings of the National Academy of Sciences, 95, 906-913.

GoLDMAN-RAKIC, P. S. (1987). Circuitry of primate prefrontal cortex and regulation of behavior by representational memory. In V. F. Plum \& V. Mountcastle (Eds.), Handbook of physiology: Vol. 5. The nervous system (pp. 373-417). Bethesda, MD: American Physiological Society.

Hays, W. L. (1994). Statistics (5th ed.). Orlando, FL: Harcourt Brace College Publishers.

JACOBY, L. L. (1991). A process dissociation framework: Separating automatic from intentional uses of memory. Journal of Memory \& Language, 30, 513-541.

JACOBY, L. L., \& BARTZ, W. H. (1972). Rehearsal and transfer to LTM. Journal of Verbal Learning \& Verbal Behavior, 11, 561-565.
JAcoby, L. L., \& Kelley, C. M. (1991). Unconscious influences of memory: Dissociations and automaticity. In A. D. Milner \& M. D. Rugg (Eds.), Foundations of neuropsychology: The neuropsychologyof consciousness (pp. 201-233). London: Academic Press.

JAMES, W. J. (1983). The principles of psychology. Cambridge, MA: Harvard University Press. (Original work published 1890)

Konishi, S., Wheeler, M. E., Donaldson, D. I., \& Buckner, R. L. (2000). Neural correlates of episodic memory retrieval mode. NeuroImage, 12, 276-286.

KuČera, H., \& Francis, W. N. (1967). Computational analysis of present-day American English. Providence, RI: Brown University Press.

LePage, M., Ghaffar, O., Nyberg, L., \& Tulving, E. (2000).Prefrontal cortex and episodic memory retrieval mode. Proceedings of the $\mathrm{Na}$ tional Academy of Sciences, 97, 506-511.

McDermott, K. B., Jones, T. C., Petersen, S. E., Lageman, S. K., \& Roediger, H. L., III (2000). Retrieval success is accompanied by enhanced activation in anterior prefrontal cortex during recognition memory: An event-related fMRI study. Journal of Cognitive Neuroscience, 12, 965-976.

Mishrin, M. (1982). A memory system in the monkey. Philosophical Transactions of the Royal Society of London: Series B, 298, 85-95.

Mugler, J. P., III, \& Brookeman, J. R. (1990). Three-dimensional magnetization-prepared rapid gradient-echo imaging (3-D MP-RAGE). Magnetic Resonance in Medicine, 15, 152-157.

Nyberg,L., Forkstam, C., Petersson, K. M., Cabeza, R., \& Ingvar, M. (2002). Brain imaging of human memory systems: Between-systems similarities and within-system differences. Cognitive Brain Research, 13, 281-292.

Ollinger,J. M., \& McAvoy, M. P. (2000, June). A homogeneitycorrection for post-hoc ANOVAs in fMRI. Poster presented at the 6th Annual Meeting of the Organization for Human Brain Mapping, San Antonio, TX.

Owen, A. M. (1997). The functional organization of working memory processes within human lateral frontal cortex: The contribution of functional neuroimaging. European Journal of Neuroscience, 9, 1329-1339.

Petrides, E., \& Milner, B. (1982). Def icits on subject-ordered tasks after frontal- and temporal-lobe lesions in man. Neuropsychologia, 20, 249-262.

Poldrack, R. A., Wagner, A. D., Prull, M. W., Desmond, J. E., Glover, G. H., \& Gabrieli, J. D. E. (1999). Functional specialization for semantic and phonological processing in the left inferior prefrontal cortex. NeuroImage, 10, 15-35.

Ranganath, C., \& D'Esposito, M. (2001). Medial temporal lobe activity associated with active maintenance of novel information. Neuron, 31, 865-873.

Ranganath, C., Johnson, M. K., \& D’Esposito, M. (2003). Prefrontal activity associated with working memory and episodic long-term memory. Neuropsychologia, 41, 378-389.

Rypma, B., Prabhakaran, V., Desmond, J. E., Glover, G. H., \& Gabrieli, J. D. E. (1999). Load-dependent roles of frontal brain regions in the maintenance of working memory. NeuroImage, 9, 216-226.

Scoville, W. B., \& Milner, B. (1957). Loss of recent memory after bilateral hippocampal lesions. Journal of Neurology, Neurosurgery, \& Psychiatry, 20, 11-21.

Shimamura, A. P., Janowsky, J. S., \& Seuire, L. R. (1991). What is the role of frontal lobe damage in memory disorders? In H. S. Levin \& H. M. Eisenberg (Eds.), The role of the frontal lobes in memory (pp. 173195). London: Oxford University Press.

SMith, E E., \& JonidEs, J. (1997). Working memory: A view from neuroimaging. Cognitive Psychology, 33, 5-42.

SMith, E. E. \& JonidES, J. (1998). Neuroimaging analyses of human working memory. Proceedings of the National Academy of Sciences, 95, 12061-12068.

SMITH, E. E., \& Jonides, J. (1999). Storage and executive processes in the frontal lobes. Science, 283, 1657-1661.

SNY DER, A. Z (1996). Difference image versus ratio image error function forms in PET-PET realignment. In R. Myers, V. Cunningham, D. Bailey, $\&$ T. Jones (Eds.), Quantification of brain function using PET (pp. 131137). San Diego: Academic Press.

Sternberg, S. (1966). High-speed scanning in human memory. Science, 153, 652-654.

Sternberg, S. (1967). Retrieval of contextual information from memory. Psychonomic Science, 8, 55-56. 
Talairach, J., \& Tournoux, P. (1988). Co-planar stereotaxic atlas of the human brain: 3-Dimensional proportional system. An approach to cerebral imaging (M. Rayport, Trans.). New York: Thieme.

Thompson-Schill, S. L., D'Esposito, M., Aguirre, G. K., \& Farah, M. J. (1997). Role of left inferior prefrontal cortex in retrieval of semantic knowledge: A re-evaluation. Proceedings of the National Academy of Sciences, 94, 14792-14797.

VAN ESSEN, D. C. (2002a). Surface management system [Computer database of surface-based atlases for the macaque and human cerebral cortex]. Retrieved November 14, 2002 from http://pulvinar.wustl. edu:8081/sums/search.do?filename $=$ ATLAS.

VAN ESSEN, D. C. (2002b). Windows on the brain: The emerging role of atlases and databases in neuroscience. Current Opinions in Neurobiology, 12, 574-579.

Van Essen, D. C., Dickson, J., Harwell, J., Hanlon, D., Anderson, C. H., \& DruRY, H. A. (2001). An integrated software system for surface-based analyses of cerebral cortex. Journal of the American Medical Informatics Association, 41, 1359-1378.

Van Essen, D. C., Drury, H. A., Harwell, J., \& Hanlon, D. (2002). CARET: Computerized anatomical reconstruction and editing toolkit [Computer software and manual]. Retrieved November 14, 2002 from http://brainmap.wustl.edu/caret.

Vargha-Khadem, F., Gadian, D. G., Watkins, K. E., Connelly, A. Van Paesschen, W., \& Mishrin, M. (1997). Differential effects of early hippocampal pathology on episodic and semantic memory. Science, 277, 376-380.

WAGNER, A. D. (1999). Working memory contributions to human learning and remembering. Neuron, 22, 19-22.
Wagner, A. D., Poldrack, R. A., Eldridge, L. L., Desmond, J. E., Glover, G. H., \& Gabrieli, J. D. E. (1998). Material-specific lateralization of prefrontal activation during episodic encoding and retrieval. NeuroReport, 9, 3711-3717.

Wagner, A. D., Schacter, D., Rotte, M., Koutstaal, W., Maril, A., Dale, A. M., Rosen, B. R., \& Buckner, R. L. (1998). Building memories: Remembering and forgetting of verbal experiences as predicted by brain activity. Science, 281, 1188-1191.

WaUgh, N. C., \& Norman, D. A. (1965). Primary memory. Psychological Review, 72, 89-104.

WiLson, M. (1988). MRC Psycholinguistic Database: Machine-usable dictionary, version 2.00. Behavior Research Methods, Instruments, \& Computers, 20, 6-10.

Woods, R. P., Cherry, S. R., \& Mazziotta, J. C. (1992). Rapid automated algorithm for aligning and reslicing PET images. Journal of Computer Assisted Tomography, 16, 620-630.

Woods, R. P., Grafton, S. T., Holmes, C. J., Cherry, S. R. \& MazZIOTTA, J. C. (1998). Automated image registration: I. General methods and intrasubject, intramodality validation. Journal of Computer Assisted Tomography, 22, 139-152.

Zola-Morgan, S., Squire, L. R., \& Amaral, D. G. (1986). Human amnesia and the medial temporal region: Enduring memory impairment following a bilateral lesion limited to field CA1 of the hippocampus. Journal of Neuroscience, 6, 2950-2967.

(Manuscript received December 10, 2002; revision accepted for publication June 2, 2003.) 